\title{
Avaliação de tecnologia educativa para crianças com diabetes: estudo metodológico
}

\author{
Evaluation of educational technology for children with type I diabetes mellitus: methodological study \\ Evaluación de tecnología educativa para niños con diabetes mellitus tipo l: estudio metodológico
}

Anna Luísa Torres Ribeiro ${ }^{1}$ (D) Éverton Fernandes de Araújo ${ }^{1}$ (C) Isla Vitória Oliveira Sousa de Pinho ${ }^{1}$ (1)

Manuela Costa Melo ${ }^{1}$ (D)

Ruth Geralda Germana Martins ${ }^{2}$ (D)

Caren Castelar Queiroz Lara $^{3}$ (C)

1. Escola Superior de Ciências da Saúde. Brasília, Distrito Federal, Brasil.

2. Secretaria de Estado de Saúde do Distrito Federal. Brasília, Distrito Federal, Brasil.

3. Secretaria de Estado de Educação do Distrito Federal. Brasília, Distrito Federal, Brasil.
Autor correspondente:

Éverton Fernandes de Araújo.

E-mail: evertonescs10@gmail.com.

Recebido em 27/07/2020.

Aprovado em 03/07/2021.

DOI:https://doi.org/10.1590/2177-9465-EAN-2020-0282

\section{Resumo}

Objetivos: descrever o processo de elaboração e avaliação de tecnologia educativa destinada à promoção do cuidado de crianças com diabetes. Método: pesquisa metodológica desenvolvida em duas fases: elaboração e avaliação. A fase de elaboração compõe: revisão de literatura; diagnóstico situacional e elaboração das ilustrações; layout; design e textos. Aplicou-se a escala Likert para a avaliação de conteúdo. Os dados foram analisados por meio do coeficiente alfa de Cronbach, Índice de Validade de Conteúdo e nível de concordância. Resultados: a definição da temática teve como base as demandas percebidas no diagnóstico situacional e a revisão de literatura. A seleção das imagens foi realizada por meio de fotografias, banco de imagens e Illustrator. As informações foram construídas como história em quadrinhos com o auxílio de designer gráfico. A tecnologia, avaliada por 12 profissionais de saúde, demonstrou nível de confiabilidade satisfatório conforme o coeficiente alfa de Cronbach $(0,7121)$. O Índice de Validade de Conteúdo $(0,875)$ e o nível de concordância $(91,67)$ foram classificados como altos. Conclusão: este estudo apresentou considerações relevantes na promoção do cuidado e no aumento do escopo de possibilidades de intervenções do profissional de saúde na perspectiva do cuidado centrado na criança e na família.

Palavras-chave: Educação em Saúde; Tecnologia Educacional; Diabetes Mellitus; Enfermagem Pediátrica; Criança.

\begin{abstract}
Objectives: to describe the process of development and evaluation of an educational technology aimed at promoting the care of children with diabetes. Method: a methodological research developed in two phases: elaboration and evaluation. The elaboration phase comprises: literature review; situational diagnosis and elaboration of illustrations; layout; design and texts. A Likert scale was applied for content evaluation. The data were analyzed using Cronbach's alpha coefficient, Content Validity Index, and level of agreement. Results: The definition of the theme was based on the demands perceived in the situational diagnosis and literature review. The selection of images was made through photographs, image banks, and Illustrator. The information was built as a comic book with the help of a graphic designer. The technology, evaluated by 12 health professionals, showed a satisfactory level of reliability according to Cronbach's alpha coefficient (0.7121). The Content Validity Index (0.875) and the level of agreement (91.67) were classified as high. Conclusion: this study presented relevant considerations in promoting care and increasing the scope of possibilities of health professional interventions from the perspective of child- and family-centered care.
\end{abstract}

Keywords: Health Education; Educational Technology; Diabetes Mellitus; Pediatric Nursing; Child.

\section{Resumen}

Objetivos: describir el proceso de elaboración y evaluación de tecnología educativa orientada a promover la atención de niños con diabetes. Método: investigación metodológica desarrollada en dos fases: elaboración y evaluación. La fase de elaboración comprende: revisión de la literatura; diagnóstico situacional y elaboración de las ilustraciones; layout; design y textos. Se aplicó la Escala Likert para evaluar el contenido. Los datos se analizaron mediante el coeficiente alfa de Cronbach, el índice de validez de contenido y el nivel de acuerdo. Resultados: la definición del tema se basó en las demandas percibidas en el diagnóstico situacional y revisión de la literatura. La selección de imágenes se realizó a través de fotografías, banco de imágenes e Illustrator Las informaciones se construyeron como un cómic con la ayuda de un diseñador gráfico. La tecnología, evaluada por 12 profesionales de la salud, mostró un nivel satisfactorio de confiabilidad, según el coeficiente alfa de Cronbach $(0,7121)$. El índice de validez de contenido $(0,875)$ y el nivel de acuerdo $(91,67)$ se clasificaron como altos. Conclusión: este estudio presentó consideraciones relevantes en la promoción del cuidado y un aumento en el alcance de las posibilidades de intervención por parte del profesional de la salud, desde la perspectiva del cuidado centrado en el niño y en la familia.

Palabras clave: Educación en Salud; Tecnología Educacional; Diabetes Mellitus; Enfermería pediátrica; Niño. 


\section{INTRODUÇÃO}

Dentre os dez países com maior número de casos de Diabetes Mellitus tipo 1 em crianças abaixo de 14 anos, o Brasil está em terceiro lugar, com $30.900 \operatorname{casos}^{1-2}$. O distúrbio afeta majoritariamente crianças e adolescentes que, desde o recebimento do diagnóstico, precisam receber informações para construir conhecimento sobre o processo saúde-doença e promover o autocuidado.

O Diabetes Mellitus tipo 1 traz grandes repercussões no cotidiano familiar e no crescimento e desenvolvimento da criança ${ }^{3}$. Diante disso, faz-se necessária a conscientização acerca do manejo da doença, que envolve hábitos de vida saudáveis, administração de insulina e monitorização da glicemia. Tendo em vista a complexidade da doença, o profissional de saúde que presta o cuidado à criança com diabetes percebe a necessidade de utilizar tecnologias educativas que facilitem o processo de educação em saúde de indivíduos, familiares e cuidadores para o entendimento do processo saúde-doença ${ }^{4}$.

Em virtude disso, a educação em saúde é reconhecida como um mecanismo eficaz na capacitação para o autocuidado, além de desenvolver o juízo crítico e a capacidade de intervenção sobre as suas próprias vidas, sendo ferramenta útil a ser utilizada no processo de ensino que compõe a assistência e, em especial, na Enfermagem Pediátrica ${ }^{4-6}$. Constata-se que a Tecnologia Educativa é um instrumento que estabelece uma relação entre a aprendizagem e a prática por meio de técnicas, oficinas, cartilhas e meios tecnológicos e possui o objetivo de auxiliar o profissional de saúde a obter melhores resultados no seu processo de trabalho, nos serviços prestados ao indivíduo e na educação e saúde ${ }^{6}$.

Portanto, este estudo justifica-se pelo fato de avançar na produção de tecnologias educativas, que podem ser implementadas por profissionais de saúde no desenvolvimento de ações para a promoção e prevenção na saúde e, assim, reduzir os índices de morbimortalidade e os custos com internações e serviços ambulatoriais no sistema nacional de saúde ${ }^{4-6}$. Além disso, possuem potencial para a inovação de processo, pois envolvem a aplicação de estratégias, referenciais e métodos conhecidos, mas que, sob a perspectiva da integração e interdisciplinaridade, são inovadores, vislumbram e favorecem o rigor e a diminuição de vieses nos resultados.

Dessa maneira, a fim de promover o autocuidado e o apoio familiar necessários ao manejo do Diabetes Mellitus tipo 1, um grupo de pesquisadores, estudantes e docentes da Escola Superior em Ciências da Saúde, em parceria com os pesquisadores do Grupo de Pesquisa na Saúde da Criança e do Adolescente (GPESCA), desenvolveu o projeto "Construção, validação e implementação de tecnologia educativa para crianças com Diabetes Mellitus Tipo 1". O grupo constatou a necessidade de utilizar uma tecnologia educativa que pudesse ser útil e fácil de ser aplicada às crianças com Diabetes Mellitus e, assim, facilitar a adesão às suas práticas de autocuidado.

Diante disso, levantou-se o seguinte questionamento: "Qual o processo percorrido para a elaboração e avaliação de um material educativo de qualidade com informações confiáveis e vocabulário claro para, assim, permitir o entendimento fácil de seu conteúdo por crianças com diabetes?". Desse modo, estabeleceu-se o objetivo deste estudo: descrever o processo de elaboração e avaliação de uma tecnologia educativa destinada à promoção do cuidado de crianças com diabetes.

\section{MÉTODO}

\section{Tipo do Estudo}

Trata-se de uma pesquisa metodológica para a elaboração e avaliação de tecnologia educativa desenvolvida em duas grandes fases: elaboração e avaliação. A fase de elaboração compõe três subfases: revisão de literatura; diagnóstico situacional e elaboração das ilustrações, layout, design e textos. A fase da avaliação compõe a avaliação de conteúdo e o teste piloto ${ }^{7}$. A subfase teste piloto com o público-alvo ainda será desenvolvida em estudo futuro após as adequações sugeridas pelos especialistas.

\section{Fase da elaboração da tecnologia educativa}

Após concretizada a sistematização do conteúdo no intuito de escolher o tema e identificar as necessidades das crianças com diabetes, foram realizados a revisão de literatura e o diagnóstico situacional. Essas duas fases foram efetivadas de maneira consecutiva e desenvolvidas entre novembro de 2017 a fevereiro de 2018.

\section{Subfase: revisão de literatura}

Por meio da aplicação da estratégia $\mathrm{PIO}$, o acrônimo para $\mathrm{P}$ (População) - Crianças; I (Intervenção) - tecnologia educativa; O (Resultados) - promoção do autocuidado, foi formulada a questão da revisão: "Quais informações a tecnologia educativa para a promoção do autocuidado para crianças com diabetes deve conter?".

Em seguida, foram utilizadas as bases de dados Biblioteca Digital de Teses e Dissertações - da Universidade de São Paulo (BDTD-USP), Coordenação de Aperfeiçoamento de Pessoal de Nível Superior (CAPES), Instituto Brasileiro de Informação e Ciência e Tecnologia (IBICT), Medical Literature Analysis and Retrieval System Online (MEDLINE), Literatura Latino-Americana em Ciências da Saúde (LILACS) e Base de Dados de Enfermagem (BDENF). Foram incluídos os estudos publicados nos idiomas português, inglês e espanhol e excluídos os editoriais, as cartas ao editor, os comentários críticos e livros abordando o assunto, assim como os artigos não disponíveis na íntegra.

Os descritores controlados foram identificados no Banco de Descritores em Medical Subject Headings (MESH) e em Ciências da Saúde (DeCS). Utilizaram-se os operadores booleanos "AND" e "OR" para realizar os cruzamentos e sistematizar a coleta da amostra. Aplicaram-se os descritores "material de ensino", "educação em saúde", "avaliação de tecnologias em saúde" e "materiais educativos e de divulgação". Utilizou-se o descritor controlado "doença crônica" associado por meio do operador booleano AND aos descritores supracitados.

Dos estudos selecionados, foi realizada a coleta de informações na qual foram extraídas as variáveis: ano de publicação; objetivo do estudo; população; tipo do estudo e a síntese dos principais resultados. 


\section{Subfase: diagnóstico situacional}

Foi realizada a pesquisa de abordagem na investigação qualitativa por estudantes de Enfermagem da Escola Superior em Ciências da Saúde por meio do Programa Institucional de Bolsas de Iniciação Científica. A pesquisa intitulada "Autorrelato da criança e adolescente no seu cotidiano com a diabetes mellitus: estudo narrativo" buscou compreender o cotidiano de crianças e adolescentes com diagnóstico de Diabetes Mellitus tipo 1. Na coleta dos dados, desenvolveram-se entrevistas semiestruturadas com 16 crianças e adolescentes atendidas em um serviço público do Distrito Federal especializado no atendimento a adultos e crianças com doenças crônicas não transmissíveis. A pesquisa foi desenvolvida entre novembro de 2017 a fevereiro de 2018.

As narrativas coletadas foram analisadas por meio do método de Análise de Conteúdo de Bardin em que foram identificadas quatro categorias temáticas: significância do Diabetes Mellitus para as crianças e os adolescentes; sentimento relacionado às mudanças ocasionadas pelo Diabetes Mellitus; aspectos relacionados aos hábitos de vida e alterações significativas para a vida, que serviram de apoio na identificação das necessidades desse grupo e nos aspectos relevantes para a elaboração da tecnologia. Nessa pesquisa, concluiu-se que o Diabetes Mellitus tipo 1 gera mudanças no contexto familiar, social e no estilo de vida e, com isso, há grande impacto psicológico à criança e ao adolescente. Por isso, faz-se necessário que o profissional de Enfermagem possa identificar os desdobramentos dessa doença em crianças e adolescentes e apresente-se como parte da rede de apoio a eles.

\section{Subfase: elaboração da tecnologia}

Após a escolha do conteúdo e a identificação das necessidades das crianças com diabetes, seguiu-se para a elaboração da tecnologia e, assim, foram escolhidos as ilustrações, o layout, o design e a composição do conteúdo. Esta fase foi desenvolvida entre março de 2019 a março de 2020.

A elaboração do texto direciona-se ao público infantil e alicerçou-se no referencial teórico do Construtivismo Piagetiano, que descreve o desenvolvimento cognitivo nas seguintes fases: sensório-motor (zero a dois anos); pré-operacional (dois a sete anos); operatório-concreto (oito a 11 anos) e operacional-formal (acima de 12 anos) ${ }^{8}$. A utilização desse referencial justifica-se pelas características específicas de cada fase do desenvolvimento infantil, o qual norteia o processo de construção da tecnologia educacional. Assim, ele foi aplicado para crianças no período do operatório-concreto.

Para o conteúdo da tecnologia educativa, foram utilizados os seguintes referenciais teóricos: National Standards for Diabetes Self-Management Education and Support ${ }^{1}$; Pocketbook for management of diabetes in childhood and adolescence in under-resourced countries - Internacional Society for Pediatric and Adolescente Diabetes ${ }^{9}$; Diretrizes 2017-2018 da Sociedade Brasileira de Diabetes ${ }^{10}$.

Para a produção da tecnologia, foram realizadas a edição e a diagramação, obedecendo aos critérios relacionados ao conteúdo, estrutura, linguagem, layout, design, sensibilidade cultural e adequação à criança com diabetes. A escolha e a criação das imagens foram realizadas por especialista em designer gráfico por meio dos aplicativos Adobe Photoshop, versão 20.0.6, e Illustrator, versão 23.0.6, e a diagramação e a composição do layout desenvolvidas no programa Illustrator.

\section{Fase da avaliação da tecnologia educativa}

\section{População do estudo}

Tratou-se de uma amostra de conveniência. Para a realização do processo de avaliação de conteúdo, foram escolhidos juízes especialistas que seguiram os critérios de inclusão e exclusão. Os critérios de inclusão foram profissionais de saúde que desenvolvem ações de prevenção e/ou promoção da saúde a crianças com diabetes há mais de cinco anos ou realizam pesquisas de validação de tecnologias educativas. Os critérios de exclusão foram profissionais de saúde que não estivessem disponíveis para participar da leitura e avaliação ou profissionais ausentes por licença, de qualquer natureza, ou férias no momento da coleta de dados.

\section{Coleta de dados e variáveis do estudo}

A coleta de dados foi realizada por meio de questionário para identificar a caracterização sociodemográfica dos participantes, apresentados as seguintes variáveis: idade; sexo; profissão; tempo de formação; área e tempo de atuação; grau de titulação e, por último, realizar a avaliação de conteúdo da tecnologia desenvolvida.

Para a avaliação de conteúdo, foi utilizada a escala tipo Likert na qual os participantes especificaram o seu nível de concordância (discordo, concordo parcialmente ou concordo) para os 14 itens apresentados no Quadro 1.

\section{Análise dos dados}

Para a avaliação das respostas, foram utilizados: o coeficiente alfa de Cronbach; o Índice de Validade de Conteúdo (IVC) e o nível de concordância ${ }^{11-14}$. Para a tabulação e o cálculo das médias do IVC, utilizou-se o programa estatístico Statistical Package for Social Sciences, versão 20.0 (SPSS), e os resultados foram apresentados por meio de estatística descritiva.

O nível de confiabilidade e a consistência de um questionário são adequados quanto mais perto de um estiver o valor da estatística, sendo aceitáveis valores de alfa que variam, em geral, entre 0,70 e $0,95^{12}$. O valor de IVC aceitável é 0,78 ${ }^{12-13}$. O IVC emprega uma escala tipo Likert com pontuação para avaliar a relevância/representatividade. Ao usar o método Porcentagem de Concordância, o percentual mínimo deve ser de $80 \%$ para ser considerado satisfatório ${ }^{14-15}$.

\section{Aspectos éticos}

O projeto foi submetido ao Comitê de Ética em Pesquisa da Secretaria de Estado de Saúde do Distrito Federal (CEP SES/DF). Toda a pesquisa foi pautada na Resolução nำ 466/12, que garante a transparência do processo e a privacidade dos participantes. Asseguraram-se a privacidade e o sigilo dos envolvidos, sendo-Ihes garantido o direito de desistência da 
Quadro 1. Avaliação dos profissionais da tecnologia educativa com relação ao conteúdo. Brasília, DF, Brasil, 2019.

\begin{tabular}{|c|c|c|c|c|}
\hline & & Discordo & $\begin{array}{l}\text { Concordo } \\
\text { parcialmente }\end{array}$ & Concordo \\
\hline 1 & $\begin{array}{l}\text { A linguagem utilizada na tecnologia educativa facilitou a } \\
\text { leitura e a interpretação das informações. }\end{array}$ & 0 & 2 & 10 \\
\hline 2 & A apresentação do layout estava clara. & 1 & 4 & 7 \\
\hline 3 & $\begin{array}{l}\text { As ilustrações estavam relevantes e adequadas ao público } \\
\text { infantil. }\end{array}$ & 0 & 4 & 8 \\
\hline 4 & $\begin{array}{l}\text { A caracterização dos personagens e a história estavam } \\
\text { claras e de fácil compreensão ao público infantil. }\end{array}$ & 0 & 2 & 10 \\
\hline 5 & $\begin{array}{l}\text { O assunto tratado estava coeso e coerente com a teoria } \\
\text { sobre a diabetes. }\end{array}$ & 0 & 2 & 10 \\
\hline 6 & $\begin{array}{l}\text { O assunto estava focado no propósito de educação em } \\
\text { saúde. }\end{array}$ & 0 & 0 & 12 \\
\hline 7 & $\begin{array}{l}\text { O assunto contempla satisfatoriamente os pontos principais } \\
\text { do tratamento de diabetes. }\end{array}$ & 0 & 1 & 11 \\
\hline 8 & O conteúdo estava de fácil compreensão. & 0 & 1 & 11 \\
\hline 9 & O conteúdo da tecnologia está adequado ao público-alvo. & 0 & 1 & 11 \\
\hline 10 & O propósito da tecnologia educativa está evidente. & 0 & 2 & 10 \\
\hline 11 & $\begin{array}{l}\text { A tecnologia educativa facilita a educação em saúde } \\
\text { realizada pelo profissional para com o paciente. }\end{array}$ & 0 & 0 & 12 \\
\hline 12 & $\begin{array}{l}\text { A tecnologia educativa tem relevância para o processo de } \\
\text { aprendizagem da criança sobre o tratamento de DM tipo } 1 .\end{array}$ & 0 & 0 & 12 \\
\hline 13 & A aplicação da tecnologia educativa é prática. & 0 & 1 & 11 \\
\hline 14 & $\begin{array}{l}\text { Seria oportuno propagar o uso desta tecnologia para } \\
\text { outros serviços de saúde. }\end{array}$ & 0 & 0 & 12 \\
\hline
\end{tabular}

Adaptado: Góes, FSN. Desenvolvimento e avaliação de objeto virtual de aprendizagem interativo sobre o raciocínio diagnóstico em Enfermagem aplicado ao recém-nascido pré-termo. Ribeirão Preto. Tese [doutorado] - Escola de Enfermagem de Ribeirão Preto. Universidade de São Paulo, 2010.

participação a qualquer momento, sem causar prejuízo de qualquer natureza para os mesmos.

No desenvolvimento das duas primeiras fases, diagnóstico situacional e revisão de literatura, o projeto foi aprovado pelo Comitê de Ética em Pesquisa da Secretaria de Estado de Saúde do Distrito Federal (CEP - SES/DF) sob o Protocolo no 2.166.881. Para o desenvolvimento das outras fases, elaboração e avaliação da tecnologia, o projeto foi aprovado pelo mesmo Comitê CEP - SES/DF e recebeu o Protocolo no 3.755.416.

\section{RESULTADOS}

\section{Elaboração da tecnologia}

A definição da temática teve, como base, as demandas de necessidades de aprendizagem percebidas nas fases de diagnóstico situacional e da revisão de literatura: definição da doença; fisiopatologia da doença (sintomas, complicações crônicas e agudas, controle da glicemia, prognóstico, medicamentos); atividades físicas (importância do exercício e contraindicações); alimentação (orientação alimentar, dificuldade em seguir as orientações sobre alimentação saudável e periodicidade das refeições) e autonomia da criança.

Essas demandas foram identificadas por estarem relacionadas às readequações na rotina diária da criança e, consequentemente, à necessidade de aprender e desenvolver habilidades para o autocuidado. O conteúdo textual foi elaborado com o objetivo de ser rico em informações, ser claro e sucinto, visto que materiais muito extensos se tornam cansativos. Todo o processo de elaboração atentou-se para a adequação da linguagem e das ilustrações de modo a facilitar a compreensão por parte da criança e sua família e aproximá-las no processo da educação em saúde. Para isso, evitouse o uso de termos técnicos e científicos e o texto foi revisado por uma pedagoga participante do grupo da pesquisa. As informações foram construídas em formato de história em quadrinhos, pois possibilita o uso de frases curtas e objetivas, as quais facilitam o entendimento e aumentam o interesse da criança pelo material.

A seleção das imagens utilizadas foi realizada por meio de fotografias da sala de uma Classe Hospitalar, em um banco de imagens gratuito, sendo elas criadas no Illustrator. O layout e a 
diagramação da página foram realizados em tamanho 15x21 cm, pensado no formato de gibi, com fontes legíveis e agradáveis à leitura e cores vivas e chamativas. Os textos foram escritos em fonte Noteworthy, tamanho seis, para balões e título, e Myriad Pro, tamanho dez para as demais informações. Utilizaram-se desenhos de linhas simples de forma a complementar e a reforçar as informações escritas. Nessa etapa, ocorreu a junção das imagens com os textos elaborados e discutidos com o apoio do grupo de pesquisadores.

O desenrolar da história ocorreu em um espaço de Classe Hospitalar, ou seja, o espaço dentro da unidade pediátrica onde um pedagogo desenvolve as atividades escolares e lúdicas com as crianças internadas. Os personagens que aparecem são: duas crianças com o diagnóstico de diabetes, um acompanhante e um profissional de Enfermagem. Uma das crianças foi recémdiagnosticada com Diabetes Melittus tipo 1 e a outra, com alguns dias de internação, demonstra, por isso, ter o conhecimento básico de sua doença. Os personagens foram desenhados para ser preenchidos e coloridos pelas crianças e seus familiares de acordo com a sua realidade. No texto, também há lacunas para o preenchimento dos nomes da criança e do acompanhante.

\section{Avaliação da tecnologia}

\section{Perfil dos participantes}

A coleta de dados foi realizada com 12 profissionais com idades entre 30 e 45 anos (58,3\%), predominantemente do sexo feminino (91,7\%). A atuação profissional foi composta por técnicos de Enfermagem (41,6\%), enfermeiros (16,6\%), médicos $(25 \%)$ e, de forma equânime, fisioterapeuta e nutricionista (8,4\%). Em relação ao grau de formação dos entrevistados, pode-se notar que a sua maioria possuía nível superior $(75 \%$ graduados), sendo que sete possuíam título de especialista (58\%). Uma parcela significativa dos profissionais entrevistados apresentava tempo de formação equivalente à experiência na área de atuação, entre 21 e 25 anos (25\%).

Todos os profissionais responderam ao questionário do tipo Likert e, para cada item da tecnologia, os profissionais avaliaram a adequação e a apresentação das informações, considerando a perspectiva dos leitores.

No Quadro 1, apresentam-se cada pergunta realizada de acordo com as suas características e a parcela de indivíduos que julgaram o item. Ao averiguar os dados do Quadro 1, verificou-se que, na avaliação de aparência, os itens 1 e 4 obtiveram, em sua maioria, conceito de concordância (83,4\%) e apenas dois profissionais admitiram o item com concordo parcialmente (16,6\%). Os itens 2 e 3 apresentaram avaliações divididas: no tópico "a apresentação do layout estava clara", sete concordaram (58,3\%) e cinco $(41,7 \%)$ concordaram parcialmente; já no tópico "as ilustrações estavam relevantes e adequadas ao público infantil", oito profissionais concordaram $(66,7 \%)$ e quatro concordaram parcialmente $(33,3 \%)$.

Na avaliação do conteúdo, o item 5 foi julgado por todos os profissionais como uma temática com propósito de educação em saúde, dados esses que revelam a adequação da tecnologia ao público-alvo. Os itens 11, 12 e 14 mostraram que todos os profissionais concordaram que a tecnologia facilita a educação em saúde e possui relevância no processo de aprendizagem da criança, além de ser oportuno o uso nos serviços de saúde. O item 13 foi julgado por apenas um profissional como "concordo parcialmente" $(8,3 \%)$ e os demais concordaram $(91,7 \%)$.

\section{Análise dos dados}

Para a avaliação do material, foram aplicadas técnicas a fim de autenticar o conteúdo do instrumento analisado. Para tanto, utilizaram-se o coeficiente alfa de Cronbach, o IVC e o nível de concordância. Diante disso, comprova-se que o questionário demonstra nível de confiabilidade satisfatória, de acordo ao coeficiente alfa de Cronbach, com resultado de 0,7121.

O IVC compreende um método muito utilizado na área de saúde em que se mede a proporção ou a porcentagem de especialistas que estão em concordância sobre determinados aspectos do instrumento e de seus itens. O IVC global obteve 0,875 e o nível de concordância das respostas foi igual a 91,67, sendo classificados como altos. Logo, a tecnologia educativa foi avaliada com êxito pelos profissionais da saúde, o que demonstra seu nível de credibilidade para aplicação.

Nenhum item do questionário foi avaliado como inadequado, assim, evidencia-se a viabilidade do material para a promoção da saúde. Entretanto, os itens que não obtiveram caráter 100\% foram adequados de acordo com as sugestões e recomendações dos juízes a fim da busca pela excelência e, dessa maneira, foram incorporadas as sugestões e recomendações. Assim, uma segunda versão da tecnologia foi submetida a outro processo de edição, revisão e diagramação (Figura 1 e Figura 2).

\section{DISCUSSÃO}

A elaboração dessa tecnologia educativa promove a educação em saúde às crianças com Diabetes Mellitus tipo 1. Por facilitar a compreensão de forma lúdica, a tecnologia possui o intuito de servir como instrumento de aproximação da realidade vivenciada pela criança e, assim, despertar o interesse e propiciar o aprendizado sobre a temática. Nesse contexto, as tecnologias educativas são relevantes, pois apresentam potencial ao permitir que o ser humano adquira conhecimento de si mesmo e do contexto em que está inserido, o que o torna capaz de entender como as próprias ações influenciam o seu padrão de saúde e exercem mudanças nesse ambiente e na sua própria conduta ${ }^{7}$. A utilização da tecnologia por uma equipe multiprofissional é também essencial para uma assistência de qualidade, além de contribuir para ações de saúde, reunindo diferentes conhecimentos especializados na temática apresentada pelo material ${ }^{16}$.

A atividade de cunho educativo deve ser realizada de forma planejada e o conhecimento do público-alvo é essencial para abordar o conteúdo educativo de acordo com a realidade, diminuindo o risco de que o material se torne incompreensível para esse público. A linguagem abordada nas tecnologias deve tornar fácil o entendimento para a população leiga, o que a torna verdadeiramente eficaz ${ }^{5}$. Assim, as informações foram escritas em forma de diálogo, pois o estilo conversacional é mais natural e 

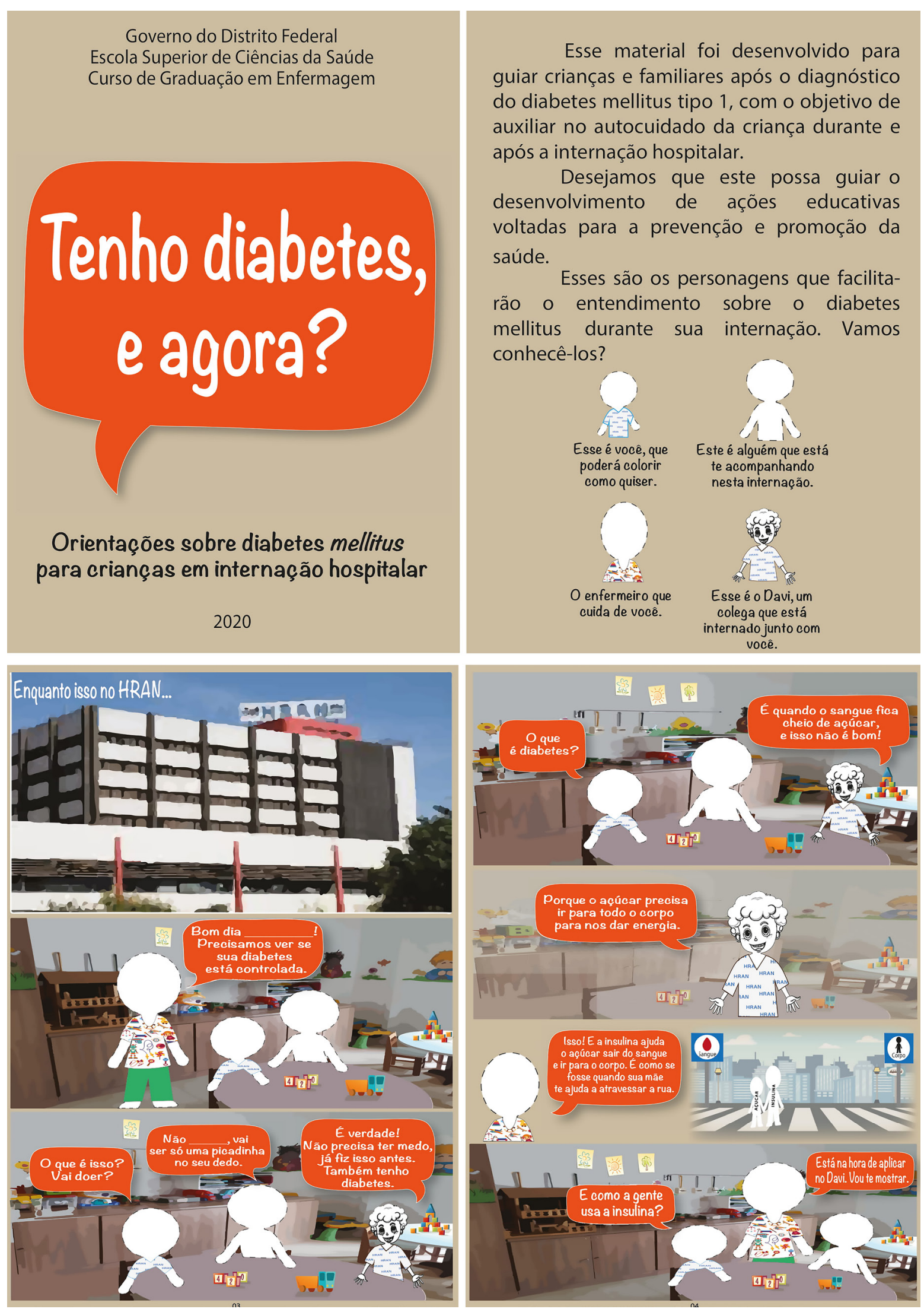

Figura 1. Tecnologia educacional

Fonte: os autores. 

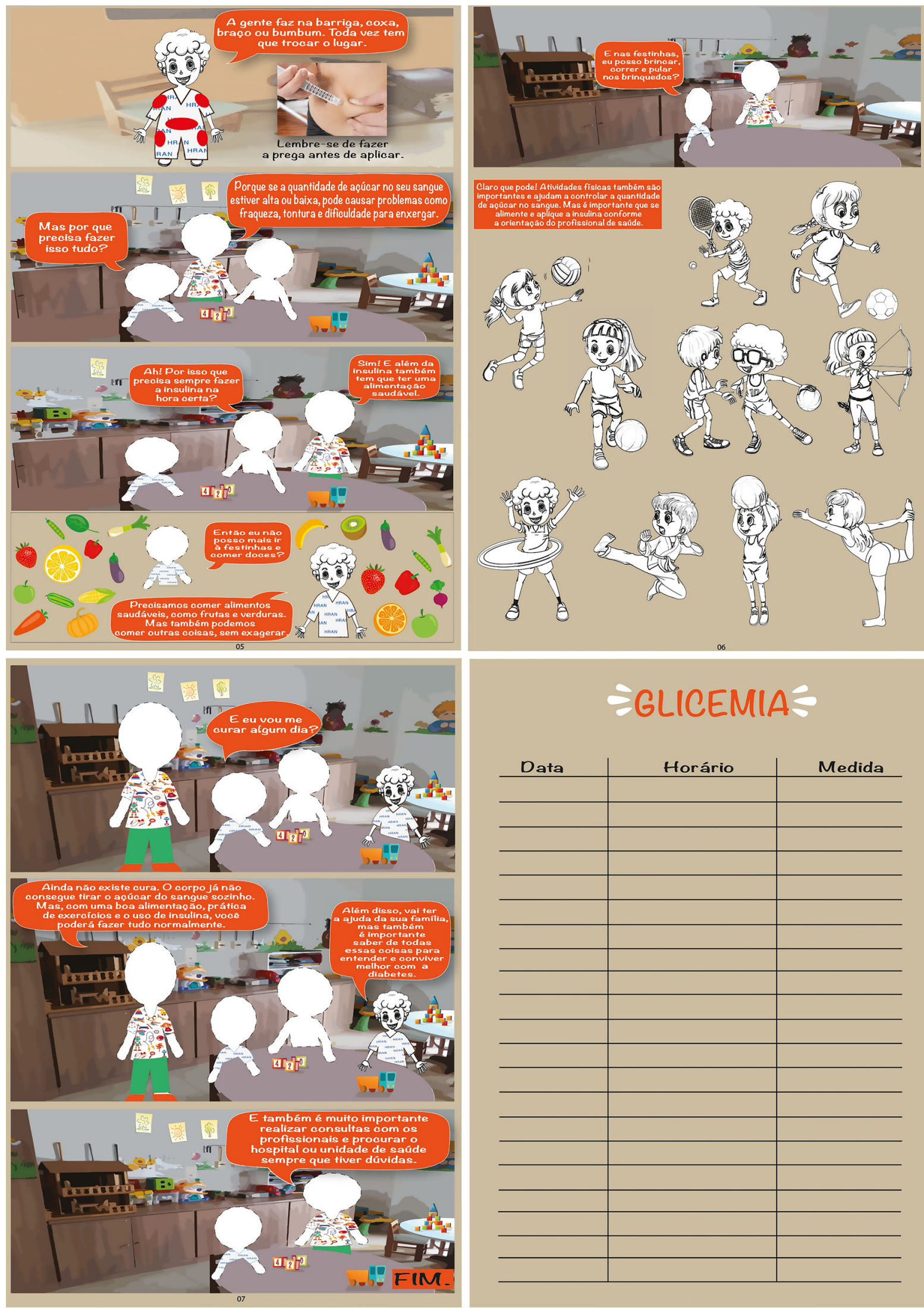

: GLICEMIA

\begin{tabular}{l|l|l} 
Data & Horário & Medida \\
\hline & & \\
\hline & & \\
\hline & & \\
\hline & & \\
\hline & & \\
\hline & & \\
\hline & & \\
\hline & & \\
\hline & & \\
\hline & & \\
\hline & & \\
\hline & &
\end{tabular}

Figura 2. Continuação da tecnologia educacional Fonte: os autores. 
fácil de ser lido e entendido. Além disso, as ilustrações elaboradas explicam ou enfatizam os aspectos e as ideias relevantes no texto.

De acordo com as fases de desenvolvimento da criança, definiram-se as crianças na fase do operatório-concreto como o público-alvo da tecnologia educativa, pois elas são capazes de realizar correlações entre o que estão vivenciando por meio da leitura e da sua realidade ${ }^{8}$. Ao unir os códigos verbais e não verbais, a estratégia de histórias em quadrinhos como recurso pedagógico pode favorecer, de maneira significativa, o processo de ensino e aprendizagem. As histórias estimulam a imaginação, desenvolvem habilidades cognitivas e são uma atividade interativa que potencializa o aprendizado ${ }^{17}$. Além disso, a possibilidade de preenchimento das lacunas e personagens contribui para a interação da história com a realidade da criança de modo a favorecer o reconhecimento da sua própria identidade.

A representação da história em uma classe hospitalar reafirma a necessidade da continuidade do processo educativo para as crianças em idade escolar, visto que esse é assegurado pela Lei $n^{\circ} 13.716$, de 24 de setembro de $2018^{18}$. A classe hospitalar assegura o direito de continuidade dos estudos e ameniza o estresse causado pela hospitalização, além de promover a educação em saúde e facilitar as interações sociais ${ }^{19}$. Esse espaço educativo tem se fortalecido quando associado à ambiência, que consiste na qualificação do espaço construído por meio de atitudes e recursos que provoquem estímulos sensoriais benéficos às pessoas ${ }^{20}$.

De modo geral, as respostas dos profissionais foram concordantes, com percentual de $91,67 \%$, o que revela a adequabilidade da tecnologia para a sua utilização. Faz-se necessário destacar os itens 1 ao 4 cujas respostas apresentaram um conceito menos aceitável. Nesses itens, os profissionais concordaram parcialmente com a apresentação do layout, o que comprova que a elaboração e a clareza do material impactam significativamente os demais critérios, influenciando, de forma direta, o emprego e o uso da tecnologia ${ }^{16}$.

Ressalta-se que, apesar de a tecnologia ter sido bem avaliada pelos especialistas, esses pontuaram observações e sugestões para as adequações do material, tais como: mudança para animações mais simples; ampliar os desenhos; utilizar desenhos grandes e coloridos; usar linguagem mais atrativa e melhorar a ordem dos quadrinhos para facilitar a compreensão da criança.

A tecnologia facilita a atuação dos profissionais de modo seguro, responsável e acessível na promoção da assistência integral na educação em saúde, contribuindo para o adequado manejo da doença e envolvendo o indivíduo no processo de aprendizagem $\mathrm{m}^{4-5}$. O material mostra-se didático, atrativo e bem estruturado e pode auxiliar no desenvolvimento de estratégias mais eficientes por parte dos profissionais de saúde, em especial, a equipe de Enfermagem, com relação aos cuidados concernentes à Diabetes Mellitus tipo 1.

A elaboração desta tecnologia configuraum avanço nas atividades de educação em saúde, uma vez que se trata de ferramenta com abordagem participativa, que pode ser utilizada por profissionais, de modo a promover o cuidado humanizado e seguro ${ }^{5,7}$. A educação em saúde é inerente ao exercício da Enfermagem, sendo reconhecida como estratégia para o enfrentamento dos múltiplos problemas de saúde que afetam a população. Dessa maneira, com a utilização da tecnologia educacional, pode-se favorecer a capacitação dos familiares e cuidadores para o correto manejo da doença e potencializar o autocuidado pelas crianças com diabetes tipo 1, de acordo com seu nível intelectual, tornando mais efetivo esse processo ${ }^{4,10}$.

\section{Relevância do estudo}

Ressalta-se a relevância deste estudo pela escassez de produções científicas na temática proposta e pela necessidade de desenvolver ações educativas relacionadas à prevenção e promoção da saúde no autocuidado de crianças com Diabetes Mellitus. Assim, pode-se facilitar o processo de trabalho do profissional, contribuindo para o adequado manejo da Diabetes Mellitus tipo 1 e promovendo a melhoria no autocuidado e na qualidade de vida dos indivíduos envolvidos. O material apresenta que nenhum item do questionário Likert foi avaliado como inadequado, o que evidencia a viabilidade do material para a promoção da saúde.

\section{CONCLUSÃO}

O papel da Enfermagem tem-se modificado ao longo dos anos para adaptar-se às mudanças cotidianas, o que reafirma a necessidade de reformulação do processo de trabalho por meio da criação de novos saberes e da readequação dos recursos tecnológicos educativos. O estudo cumpriu o objetivo proposto, que foi elaborar e avaliar uma tecnologia educativa para a promoção dos cuidados de crianças com diabetes. A elaboração dessa tecnologia educacional foi possível por ter ocorrido de maneira participativa, dialógica e coletiva de modo a evidenciar que é executável, podendo ser aplicada na elaboração de materiais educativos destinados à educação e promoção da saúde. A tecnologia educativa foi avaliada com relação ao conteúdo e atentou-se às considerações e sugestões dos profissionais envolvidos no estudo.

Espera-se que este material seja utilizado para disseminar a educação sobre a temática abordada, com foco na abordagem lúdica e compreensiva para crianças, além de promover visibilidade às pessoas e famílias que se encontram nesse contexto.

Salienta-se que esta tecnologia educativa foi produzida em um contexto para ser aplicado em uma única unidade de internação hospitalar com especificidades da realidade local. Essa limitação revela que não é possível abranger a diversidade da realidade brasileira com uma tecnologia padronizada institucionalmente. Para a possibilidade de ampliar a sua aplicação, torna-se necessário incorporar as dimensões, os valores e até mesmo os padrões de linguagem peculiares de determinadas localidades e realidades.

Isso requer a adaptação transcultural dessa cartilha ou mesmo a produção de novos materiais. Retoma-se a importância da construção coletiva, com a participação ativa dos agentes envolvidos no processo, que certamente agregará maior valor a esse recurso. Outra limitação é a fragilidade com relação ao tamanho da amostra, por tratar apenas de especialistas em saúde, sendo necessária a avaliação por crianças e familiares, como também por experts em designer gráfico.

Destaca-se que essa tecnologia recebeu a premiação na modalidade "Melhor Pesquisa de Campo" do II Congresso de 
Enfermagem da Universidade Católica de Brasília, realizado em Brasília, Distrito Federal, em novembro de 2019.

\section{FINANCIAMENTO}

Pesquisa desenvolvida mediante a concessão de Bolsa de Iniciação Científica do Conselho Nacional de Desenvolvimento Científico e Tecnológico/CNPq. Edital ํㅜㄴ, de 16 de abril de 2019, processo SEI no 00064-00000907/2019-61 da Escola Superior de Ciências da Saúde (ESCS), mantida pela Fundação de Ensino e Pesquisa em Ciências da Saúde (FEPECS).

\section{CONTRIBUIÇÕES DOS AUTORES}

Desenho do estudo. Anna Luísa Torres Ribeiro. Éverton Fernandes de Araújo. Isla Vitória Oliveira Sousa de Pinho. Manuela Costa Melo. Ruth Geralda Germana Martins. Caren Castelar Queiroz Lara

Coleta ou produção dos dados. Anna Luísa Torres Ribeiro. Éverton Fernandes de Araújo. Isla Vitória Oliveira Sousa de Pinho. Manuela Costa Melo. Ruth Geralda Germana Martins. Caren Castelar Queiroz Lara

Análise de dados. Anna Luísa Torres Ribeiro. Éverton Fernandes de Araújo. Isla Vitória Oliveira Sousa de Pinho. Manuela Costa Melo. Ruth Geralda Germana Martins. Caren Castelar Queiroz Lara

Interpretação dos resultados. Anna Luísa Torres Ribeiro. Éverton Fernandes de Araújo. Isla Vitória Oliveira Sousa de Pinho. Manuela Costa Melo. Ruth Geralda Germana Martins. Caren Castelar Queiroz Lara

Redação e revisão crítica do manuscrito. Anna Luísa Torres Ribeiro. Éverton Fernandes de Araújo. Isla Vitória Oliveira Sousa de Pinho. Manuela Costa Melo. Ruth Geralda Germana Martins. Caren Castelar Queiroz Lara

Aprovação da versão final do artigo. Anna Luísa Torres Ribeiro. Éverton Fernandes de Araújo. Isla Vitória Oliveira Sousa de Pinho. Manuela Costa Melo. Ruth Geralda Germana Martins. Caren Castelar Queiroz Lara

Responsabilidade por todos os aspectos do conteúdo e a integridade do artigo publicado. Anna Luísa Torres Ribeiro. Éverton Fernandes de Araújo. Isla Vitória Oliveira Sousa de Pinho. Manuela Costa Melo. Ruth Geralda Germana Martins. Caren Castelar Queiroz Lara

\section{EDITOR ASSOCIADO}

Candida Caniçali Primo (1)

\section{EDITOR CIENTIÍFICO}

\author{
Ivone Evangelista Cabral (1)
}

\section{REFERÊNCIAS}

1. Beck J, Greenwood DA, Blanton L, Bollinger ST, Butcher MK, Condon JE et al. National Standards for Diabetes Self-Management Education and Support. Diabetes Care. 2017;40(10):1409-19. http://dx.doi. org/10.2337/dci17-0025. PMid:28754780.

2. Flor LS, Campos MR. The prevalence of diabetes mellitus and its associated factors in the Brazilian adult population: evidence from a population-based survey. Rev Bras Epidemiol. 2017;20(1):16-29. http:// dx.doi.org/10.1590/1980-5497201700010002. PMid:28513791.

3. Sand P, Blom MD, Forsander G, Lundin CS. Family dynamics when a child becomes chronically ill: impact of type 1 diabetes onset in children and adolescentes. Nordic Psychol. 2018;70(2):97-114. http://dx.doi.or g/10.1080/19012276.2017.1362990.

4. Ortiz LMO, Damião EBC, Rossato LM, Alves RCP. Melhores práticas de enfermagem em educação em diabetes à criança hospitalizada: uma revisão integrativa. Rev. Eletr. Enf. 2017;19:a56. http://dx.doi. org/10.5216/ree.v19.45655.

5. Silva DML, Carreiro FA, Mello R. Educational technologies in nursing assistance in health education: Integrating review. Rev Enferm UFPE. 2017;11(Suppl. 2):1044-51.

6. Azevêdo AVDS, Lançoni AC Jr, Crepaldi MA. Nursing team, family and hospitalized child interaction: an integrative review. Cien Saude Colet 2017;22(11):3653-66. PMid:29211171.

7. Teixeira E, Martins TDR, Miranda PO, Cabral BG, Silva BAC, Rodrigues LSS. Educational technology on potpartum care: development and validation. Rev Baiana Enferm. 2016;30(2):1-10.

8. Cevolane L, Dantos APT, Vinco GF, Fazolo LC, Donatelli SM, Canal FD. Densenvolvimento humano: um esboco da perspectiva de Jean Piaget. Rev Dimensão Acadêmica. 2017;2(1):63-78.

9. International Diabetes Federation, International Society for Pediatric and Adolescente Diabetes. Pocketbook for management of diabetes in childhood and adolescence in under-resourced countries [Internet]. Tallahassee: Life for a Child; 2017 [citado 2020 jan 05]. Disponível em: https://lifeforachild. org/education-resources/guidelines/57:pocketbook-for-management-ofdiabetes-in-childhood-and-adolescence-in-under-resourced-countries.html.

10. Sociedade Brasileira de Diabetes. Diretrizes Sociedade Brasileira de Diabetes. 2017-2018. São Paulo: Editora Científca Clannad; 2018 [citado 2020 jan 05]. Disponível em: https://www.diabetes.org.br/profissionais/ images/2017/diretrizes/diretrizes-sbd-2017-2018.pdf

11. Mierzejewska E, Honorato-Rzeszewicz T, Świątkowska D, Jurczak Czaplicka M, Maciejewski T, Fijałkowska A et al. Evaluation of questionnaire as an instrument to measure the level of nutritional and weight gain knowledge in pregnant women in Poland. A pilot study. PLoS One. 2020;15(1):e0227682. http://dx.doi.org/10.1371/journal. pone.0227682. PMid:31940402.

12. Yusoff MSB. ABC of content validation and content validity index calculation. Education in Medicine Journal. 2019;11(2):49-54. http:// dx.doi.org/10.21315/eimj2019.11.2.6.

13. Souza AC, Alexandre NMC, Guirardello ED. Psychometric properties in instruments evaluation of reliability and validity. Epidemiol Serv Saude. 2017;26(3):649-59. http://dx.doi.org/10.5123/S1679-49742017000300022. PMid:28977189.

14. Ribeiro LCC, Oliveira TC, Moreira S, Paula FA. Construction and validation of manual on burnout in teachers. Rev Enferm. 2017;7(1):1-9.

15. Silva NM, Rosado SR, Santos MA, Sonobe HM. Validation of characterization instrument for patients with colorectal pathologies. Rev Enferm UFPE. 2019;13(4):960-5.

16. Maniva SJCF, Carvalho ZMF, Gomes RKG, Carvalho REFL, Ximenes LB Freitas CHA. Educational Technologies for health education on stroke: an integrative review. Rev Bras Enferm. 2018;71(Suppl. 4):1724-31. http://dx.doi.org/10.1590/0034-7167-2017-0041. PMid:30088646.

17. Rolim KMC, Pinheiro CW, Magalhães FJ, Frota MA, Mendonça FAC Fernandes HIVM. História em quadradinhos: tecnologia em saúde para a humanização da assistência à criança hospitalizada. Rev Enf Ref 2017;IV(14):69-78. http://dx.doi.org/10.12707/RIV17028.

18. Souza ZS, Rolim CLA. As vozes das professoras na pedagogia hospitalar: descortinando possibilidades e enfrentamentos. Rev Bras Educ Espec. 2019;25(3):403-20. http://dx.doi.org/10.1590/s1413-65382519000300004.

19. Menzani RM, Regueiro EMG, Leiva JC. Ser criança na classe hospitalar: a dimensão psicológica na interface educação e saúde. Rev Bras Multidiscip. 2017;20(1):106. http://dx.doi.org/10.25061/2527-2675/ ReBraM/2017.v20i1.476.

20. Martins CP, Luzio CA. HumanizaSUS policy: anchoring a ship in space. Interface. 2017;21(60):13-22. http://dx.doi.org/10.1590/1807-57622015.0614. 